\title{
Black Holes and Black Strings on Cylinders
}

\author{
T. Harmark ${ }^{1}$, N.A. Obers ${ }^{2}$ \\ 1 Jefferson Physical Laboratory \\ Harvard University, Cambridge, MA 02138, USA \\ 2 The Niels Bohr Institute \\ Blegdamsvej 17, DK-2100 Copenhagen Ø, Denmark
}

\begin{abstract}
We review the recently discovered ansatz that describes non-extremal charged dilatonic branes of string/M-theory with a transverse circle. The ansatz involves a new coordinate system that interpolates between the spherical and cylindrical case, and reduces the equations of motion to a set of equations on one unknown function of two variables. The function is independent of the charge, so that the ansatz can also be used to construct neutral black holes on cylinders and near-extremal charged dilatonic branes with a transverse circle. The construction enables us to argue that, for sufficiently large mass, there exists a neutral solution that breaks translational invariance in the circle direction, and has larger entropy than that of the neutral black string of the same mass.
\end{abstract}

\section{Introduction}

The study of black holes on cylinders $\mathbb{R}^{d-1} \times S^{1}$ is interesting from many perspectives. While in flat space the static neutral black hole is uniquely described by the Schwarzschild solution, black holes on cylinders exhibit a more interesting dynamics and richer phase structure, as the radius of the circle introduces a macroscopic scale in the system. Moreover, $\mathbb{R}^{d-1} \times S^{1}$ has a non-trivial topology in the sense that it is non-contractible with a non-trivial fundamental group. As a consequence, the event horizon of black objects may not only have topology $S^{d-1}$ but also $S^{d-2} \times S^{1}$. Finally, black holes on cylinders show up in various contexts in string theory as branes on a transverse circle, their near-horizon limits being relevant in the AdS/CFT correspondence and Matrix theory.

If we start with a small mass black hole on a cylinder and raise its mass, so that the horizon radius increases, one will reach at some point a critical mass $M_{c}$ for which the event horizon meets itself across the cylinder. At present the following scenarios have been proposed for what happens when this point in the phase diagram is reached:

- The most standard point of view is that for $M>M_{c}$ the black will uniformly distribute its mass along the circle, forming a neutral black string with horizon topology $S^{d-2} \times S^{1}$, which is classically stable. The corresponding transition is first order.

- The second scenario which is put forward in 11 claims the existence of a new solution for $M>M_{c}$, also with horizon topology $S^{d-2} \times S^{1}$, but which is not translationally invariant along the circle (see also Ref. [2]). The phase transition involved is of higher order (second or third). This non-uniform solution has higher entropy than the corresponding black 
string of equal mass. Only in the infinite mass limit do we recover the black string. - There is another mass scale in the problem $M_{u}>M_{c}$, such that for $M_{c}<M<M_{u}$ we have a phase with a non-uniform black string and for $M>M_{u}$ a phase with a uniform black string. This scenario was put forward in [3] motivated by [4, 5]. The scenario has been considered further using numerical techniques in [6, 7].

In this talk we review the evidence for the second scenario above, as presented in Ref. 1]. The claim is supported by following an analytic approach toward constructing explicit solutions for black holes on cylinders $\mathbb{R}^{d-1} \times S^{1}$ for $d \geq 4^{1}$. As part of the construction we find a new coordinate system in which we are able to conjecture a general ansatz for non-extremal charged dilatonic branes with a transverse circle. Note here that we sometimes loosely refer to $p$-branes with $d$ transverse directions as black holes in a $(d+1)$-dimensional space-time, since the $p$ world-volume directions can be considered to be compactified.

We show that the equations of motion imply that the ansatz is in fact fully described by only one function, and present strong arguments in favor of consistency of the system. Moreover, we find that this function is independent of the charge which means that we can map the ansatz for non-extremal charged dilatonic branes with a transverse circle to an ansatz for the near-extremal limit of those and, moreover, to an ansatz for neutral black holes on cylinders. Thus, our construction applies to three classes of black holes on cylinders: the non-extremal charged dilatonic branes of String/M-theory with a transverse circle, the near-extremal limit of these branes and finally neutral black holes on cylinders.

The existence of the new solutions has the consequence that for $M>M_{c}$ we have another black object with the same horizon topology as the black string. We argue via our construction that the new solutions have larger entropy than the black strings. This means that for $M>M_{c}$ the black string can gain entropy by spontaneously breaking the translational invariance and redistributing its mass according to our new solution. We have thus found a new instability of the black string for large mass $M>M_{c}$ which is not classical in nature. The argument easily generalizes to a similar statement for the nonand near-extremal case.

To further put our result in broader context, is also instructive to consider a very massive black string on a cylinder and imagine what happens when one lowers its mass. In this case the corresponding scenarios are as follows:

- Gregory and Laflamme [9] found that a neutral black string wrapping a cylinder is classically unstable if its mass becomes sufficiently small $M<M_{\mathrm{GL}}$. Since the entropy of a black hole with the same mass is larger, it was conjectured that the black string decays to a black hole. This involves a first order transition from a black object of topology $S^{d-2} \times S^{1}$ to one of topology $S^{d-1}$.

- Horowitz and Maeda 4 argued that an event horizon cannot have a collapsing circle in a classical evolution. This obstruction for the black string to change the topology of the event horizon, lead to the conjecture [4] that there exist new classical solutions with event horizon of topology $S^{d-2} \times S^{1}$ that are non-translationally invariant along the circle whenever the black string is classically unstable. The black string thus decays to this nonuniform solution. Following the investigation of [5] and the further work of Refs. [3, 6, 7] one is then lead to the reverse process of the third bullet above.

- Finally we have the scenario advocated in Ref. [1, in which for $M>M_{c}$ there exists a non-uniform black hole solution while for $M<M_{c}$ the black hole solution is found after

\footnotetext{
${ }^{1}$ Explicit solutions have been constructed for black holes on $\mathbb{R}^{2} \times S^{1}$ [8. However, the methods used there are highly particular to that case and cannot be generalized to $\mathbb{R}^{d-1} \times S^{1}$ for $d \geq 4$.
} 
a higher order phase transition. This is of course the reverse of the second bullet above.

The focus in the present talk will be directed toward black holes on cylinders with large masses $M>M_{c}$, for which we summarize the arguments for the existence of solutions that are non-uniform and classically stable [1. Another approach to this problem can be found in Ref. [2], which uses specially constructed initial data to argue that certain classes of charged non-extremal $p$-branes have a stable non-translationally invariant solution with horizons of topology $S^{d-2} \times S^{1}{ }^{2}$

\section{New coordinates and the ansatz}

Finding solutions of black holes on the $d$-dimensional cylinder $\mathbb{R}^{d-1} \times S^{1}$ involves solving highly complicated nonlinear equations. One way to see this is by considering the covering space $\mathbb{R}^{d}$ of the cylinder, on which a black hole in $\mathbb{R}^{d-1} \times S^{1}$ is really a one-dimensional array of black holes. Since the interactions between black holes are in general non-linear, the geometry is very complicated once the back-reaction is included. Another way to see the complication is to note that neither the spherical symmetry nor the cylindrical symmetry applies in general for such a black hole solution. As a consequence one is forced to consider a solution with functions that depend on two coordinates rather than one.

Another essential ingredient in finding an appropriate ansatz is the requirement that the solution should interpolate between the usual black brane with transverse space $\mathbb{R}^{d}$, which is a good description at small mass, and the black brane smeared on the transverse circle, which is a good description at large mass. We furthermore demand that the solution should reduce to the extremal charged dilatonic $p$-branes with transverse space $\mathbb{R}^{d-1} \times S^{1}$ for zero temperature. In order to capture these features in an ansatz we must therefore find an appropriate coordinate system that can be used in both the small and large mass limits and also for the extremal solution.

In finding this coordinate system we use the extremal dilatonic $p$-brane with transverse space $\mathbb{R}^{d} \times S^{1}$ as a guide. This background involves the harmonic function

$$
H=1+\frac{L^{d-2}}{R_{T}^{d-2}} F_{d-2}\left(\frac{r}{R_{T}}, \frac{z}{R_{T}}\right), \quad F_{2 s}(a, b) \equiv \sum_{n \in \mathbb{Z}}\left(a^{2}+(2 \pi n+b)^{2}\right)^{-s}
$$

where $R_{T}$ is the size of the transverse circle, $L$ is related to the charge and $(r, z)$ are cylindrical coordinates. The notation used for the spherical coordinates is $(\rho, \theta)$. We then define a new coordinate $R(r, z)$ by

$$
R^{3-d}=k_{d}^{-1} F_{d-2}\left(\frac{r}{R_{T}}, \frac{z}{R_{T}}\right)
$$

which has the property that it interpolates between the radial variable $r$ in cylindrical coordinates for $R \gg 1$ and the radial variable in spherical coordinates $\rho=\sqrt{r^{2}+z^{2}}$ for $R \ll 1$. Here $k_{d}$ is a number that can be found in [1]. The coordinate $v$ that similarly interpolates between $z$ for $R \gg 1$ and an angle $\theta$ on the $S^{d-1}$ sphere for $R \ll 1$ is found by imposing that the flat space metric in the new coordinates $(R, v)$ is diagonal. This leads to the defining set of equations

$$
\frac{\partial v}{\partial r}=\frac{1}{(d-3) k_{d}}\left(\frac{r}{R_{T}}\right)^{d-2} \frac{\partial F_{d-2}}{\partial z}, \quad \frac{\partial v}{\partial z}=-\frac{1}{(d-3) k_{d}}\left(\frac{r}{R_{T}}\right)^{d-2} \frac{\partial F_{d-2}}{\partial r}
$$

\footnotetext{
${ }^{2}$ See also Ref. [10 for a Petrov classification of five-dimensional metrics, relevant for the construction of black holes on $R^{3} \times S^{1}$.
} 
which is integrable as a consequence of the harmonic equation $\nabla^{2} F_{d-2}=0$. Ref. [1] gives an explicit solution of these equations in terms of a series expansion. It is straightforward to write down the flat metric on the $d$-dimensional cylinder, and that of the extremal dilatonic $p$-brane in terms of the $(R, v)$ coordinates.

In order to formulate our ansatz in the new coordinate system, we first specify the boundary conditions that we want to impose. To this end we introduce a general Schwarzschild radius $R_{0}$ as the maximal value of the $R$ coordinate on the horizon. The boundary conditions are then:

(i) The solution reduces to an ordinary black $p$-brane with transverse space $\mathbb{R}^{d}$ when $R_{0} \leq R \ll 1$.

(ii) The solution reduces to a black $p$-brane smeared on the transverse circle when $R \geq$ $R_{0} \gg 1$.

(iii) For $R_{0} / R \rightarrow 0$ the solution approaches the extremal dilatonic $p$-brane with transverse space $\mathbb{R}^{d-1} \times S^{1}$.

(iv) The horizon is located at constant $R$. The rationale behind this condition is that the equipotential surfaces of the charge potential are defined by $R$ being constant and we expect the horizon to be at an equipotential surface ${ }^{3}$. Obviously, the horizon is then defined by the equation $R=R_{0}$.

Our ansatz for charged dilatonic black holes on $\mathbb{R}^{d} \times S^{1}$ is then

$$
\begin{gathered}
d s_{D}^{2}=H^{-\frac{d-2}{D-2}}\left[-f d t^{2}+\sum_{i=1}^{p}\left(d x^{i}\right)^{2}+H R_{T}^{2}\left(f^{-1} A d R^{2}+\frac{A}{K^{d-2}} d v^{2}+K R^{2} d \Omega_{d-2}^{2}\right)\right] \\
e^{2 \phi}=H^{a}, \quad A_{01 \cdots p}=\operatorname{coth} \alpha\left(1-H^{-1}\right) \\
f=1-\frac{R_{0}^{d-3}}{R^{d-3}}, \quad H=1+\frac{R_{0}^{d-3} \sinh ^{2} \alpha}{R^{d-3}}, \quad h_{d}=R_{0}^{d-3} \cosh \alpha \sinh \alpha
\end{gathered}
$$

with two undetermined functions $A(R, v)$ and $K(R, v)$ at this point. In this form, the background already satisfies the equation of motion (EOM) of the dilaton and the gauge potential, so that the only remaining non-trivial EOMs are those for the metric. It turns out that these are independent of the constant $h_{d}$ which is proportional to the charge. We can therefore map our ansatz for non-extremal charged dilatonic branes with a transverse circle to an ansatz for neutral black holes on cylinders $(H=1$ in (4) $)$ as well as nearextremal charged dilatonic branes with a transverse circle. The boundary conditions will be modified accordingly. Consequently, the problem of finding solutions of black dilatonic $p$-brane with transverse space $\mathbb{R}^{d-1} \times S^{1}$ can be mapped to that of finding neutral black holes on $\mathbb{R}^{d-1} \times S^{1}$, which we focus on in the remainder.

The EOMs are given by $R_{\mu \nu}=0$, yielding four non-trivial equations, $R_{R R}=R_{v v}=$ $R_{R v}=R_{\phi_{1} \phi_{1}}=0$ (see Eq. (6.1-4) in [1] for the explicit form). The $R_{\phi_{1} \phi_{1}}=0$ equation can be trivially solved for $A(R, v)$ so that we end up with a system of three differential equations on $K(R, v)$ containing up to four derivatives. At this point one may wonder about the consistency of the obtained system of equations. A detailed analysis in Ref. [1] shows that through second order in a large $R$ expansion these three equations are consistent. We regard this as non-trivial confirmation of our expectation that our ansatz is consistent ${ }^{4}$. Moreover, by studying the Newtonian limit, it was also shown [1 that small black holes on cylinders can be correctly incorporated in the ansatz as well.

\footnotetext{
${ }^{3}$ This holds also for spinning brane solutions (See for example [11]).

${ }^{4}$ Moreover, recently it was shown [7] that the numerical non-uniform black string solution of [5] 6] can be put into the ansatz of [1].
} 
We review here some of the salient features of the perturbative analysis. First, the boundary condition (iii) above becomes

$$
K(R, v) \rightarrow K_{(0)}(R, v) \text { for } \frac{R_{0}}{R} \rightarrow 0
$$

where $K_{(0)}(R, v)=\left(\frac{r}{R_{T}}\right)^{2}\left(\frac{F_{d-2}}{k_{d}}\right)^{\frac{2}{d-3}}$ is the corresponding function that enters the flat metric on the $d$-dimensional cylinder. This means that $K(R, v)$ should have an expansion in powers of $R_{0}^{d-3} / R^{d-3}$ with $K_{(0)}(R, v)$ being the zeroth order term. In particular, for $R \gg 1$ we then impose the boundary condition

$$
K(R, v)=1-\chi\left(R_{0}\right) \frac{R_{0}^{d-3}}{R^{d-3}}+\cdots \quad, \quad \chi\left(R_{0}\right)=\left\{\begin{array}{cll}
\frac{1}{(d-2)(d-3)} & \text { for } \quad R_{0} \ll R_{c} \\
0 & \text { for } \quad R_{0} \gg R_{c}
\end{array} .\right.
$$

Here the $R_{0} \ll R_{c}$ value of $\chi$ was obtained from the Newtonian limit of small black holes on the cylinder, while the $R_{0} \gg R_{c}$ value follows by comparison to the black string solution. By combining periodicity properties in $v$ with the "expansion parameter" $e^{-R}$ we have then been able to show through second order that, given a value of $\chi\left(R_{0}\right), K(R, v)$ is completely determined. In some sense the function $\chi\left(R_{0}\right)$ contains all physical information about the solution, for example the entire thermodynamics can be derived from it. At present we do not know the exact solution of the EOMs nor the exact form of $\chi\left(R_{0}\right)$, but we can argue the existence of solutions due to the perturbative analysis as well as on more general physical grounds. For $R_{0}>R_{c}$ these must necessarily be non-translationally invariant along the circle.

\section{$3 \quad$ Thermodynamics and stability}

Though an exact solution is so far lacking, one can still obtain a surprising amount of information on our new non-uniform solutions. First, using the Killing vector $\partial / \partial t$, we find the surface gravity on the Killing horizon, which turns out to depend on $\gamma\left(R_{0}\right) \equiv\left(\left.A\right|_{R=R_{0}}\right)^{-1 / 2}$. Evaluation of the EOMs on the horizon yields the satisfying result that $A(R, v)$ is independent of $v$ on the horizon, so that we have a well-defined constant temperature of the black hole. The thermodynamics for the neutral case is then summarized by

$$
\begin{gathered}
T=\gamma\left(R_{0}\right) \frac{d-3}{4 \pi R_{0} R_{T}}, \quad S=\frac{1}{\gamma\left(R_{0}\right)} \frac{\Omega_{d-2} 2 \pi R_{T}}{4 G_{d+1}}\left(R_{0} R_{T}\right)^{d-2} \\
M=\frac{\Omega_{d-2} 2 \pi R_{T}}{16 \pi G_{d+1}}(d-3)\left(R_{0} R_{T}\right)^{d-3}\left[\frac{d-2}{d-3}-\chi\left(R_{0}\right)\right] .
\end{gathered}
$$

Note that the first law of thermodynamics then implies a relation between $\gamma\left(R_{0}\right)$ and $\chi\left(R_{0}\right)$ which was utilized in Ref. [1] to examine the consequences of a smooth interpolation between small and large black hole solutions on cylinders. In fact, we expect this relation to specify the physical subspace of solutions by determining the $R_{0}$ dependence of $\chi$.

Further knowledge of $\chi\left(R_{0}\right)$ can be obtained using again the Newtonian limit. One considers static matter with both a mass density and negative pressure, which corresponds to the binding energy of the black hole on the cylinder. The analysis shows that $\chi$ can be interpreted directly in terms of the binding energy relative to the mass of the object. Then using the physical fact that the binding energy increases with $R_{0}$ we have shown that $\chi\left(R_{0}\right)$ is a monotonically decreasing function, interpolating between the extremal 
values given in (8), and hence always positive. By comparing the thermodynamics (9), (10) to that of the neutral black string it then follows that $\chi\left(R_{0}\right) \geq 0$ implies the entropy inequality

$$
S(M)>S_{\text {str }}(M)
$$

showing that the entropy of the non-translationally invariant solution is larger than that of the black string. The reason for comparing the entropies at a given mass, i.e. working in the microcanonical ensemble, is that we want to check whether it is thermodynamically favorable for a black string of a given mass to redistribute itself into the non-translationally invariant solution in order to gain entropy. It thus follows that this is always favorable, signaling a new instability of the black string which is quantum mechanical in nature.

\section{Further comments}

There are many issues open for further study, among which we mention a few. First it would naturally be very interesting if it were possible to obtain exact forms of our new solutions by explicitly solving the equations. Numerical study would similarly be important in order to further check some of our assumptions. More generally, it would be desirable to have a better understanding of the space of solutions for black holes on cylinders, as well as the change of topology of the horizon. It would furthermore be illuminating to study more precisely the nature of the quantum instability that causes the black string to decay to our new solutions. There are also many applications in string theory of these new non-uniform solutions. In particular the application to thermal little string theory (LST) was started in Ref. [1], one of the aims being further insight into the thermodynamic properties of LST near the Hagedorn temperature [12, 13. Finally, the

work presented here could also be relevant in the considerations of [14] about stability of near-extremal branes.

Acknowledgement NO would like to thank the organizers of the 35th Ahrenshoop meeting for an interesting workshop and the invitation to present this work.

\section{References}

[1] T. Harmark and N. A. Obers, "Black holes on cylinders," JHEP 05 (2002) 032, hep-th/0204047

[2] G. T. Horowitz and K. Maeda, "Inhomogeneous near-extremal black branes," Phys. Rev. D65 (2002) 104028, hep-th/0201241; G. T. Horowitz, "Playing with black strings," hep-th/0205069.

[3] B. Kol, "Topology change in general relativity and the black-hole black-string transition," hep-th/0206220

[4] G. T. Horowitz and K. Maeda, "Fate of the black string instability," Phys. Rev. Lett. 87 (2001) 131301, hep-th/0105111.

[5] S. S. Gubser, "On non-uniform black branes," Class. Quant. Grav. 19 (2002) 4825-4844, hep-th/0110193.

[6] T. Wiseman, "Static axisymmetric vacuum solutions and non-uniform black strings," hep-th/0209051. 
[7] T. Wiseman, "From black strings to black holes," hep-th/0211028.

[8] R. C. Myers, "Higher dimensional black holes in compactified space- times," Phys. Rev. D35 (1987) 455; A. R. Bogojevic and L. Perivolaropoulos, "Black holes in a periodic universe," Mod. Phys. Lett. A6 (1991) 369-376; H. Lu, C. N. Pope, and K. W. Xu, "Black p-branes and their vertical dimensional reduction," Nucl. Phys. B489 (1997) 264-278, hep-th/9609126; I. V. Lavrinenko, H. Lu, C. N. Pope, and T. A. Tran, "Harmonic superpositions of non-extremal p-branes," Int. J. Mod. Phys. A13 (1998) 273-294, hep-th/9702058.

[9] R. Gregory and R. Laflamme, "Black strings and p-branes are unstable," Phys. Rev. Lett. 70 (1993) 2837-2840, hep-th/9301052.

[10] P.-J. De Smet, "Black holes on cylinders are not algebraically special," Class. Quant. Grav. 19 (2002) 4877-4896, hep-th/0206106.

[11] T. Harmark and N. A. Obers, "Thermodynamics of spinning branes and their dual field theories," JHEP 01 (2000) 008, hep-th/9910036.

[12] J. M. Maldacena and A. Strominger, "Semiclassical decay of near-extremal fivebranes," JHEP 12 (1997) 008, hep-th/9710014 J. M. Maldacena, "Statistical entropy of near extremal five-branes," Nucl. Phys. B477 (1996) 168-174, hep-th/9605016.

[13] T. Harmark and N. A. Obers, "Hagedorn behaviour of little string theory from string corrections to NS5-branes," Phys. Lett. B485 (2000) 285, hep-th/0005021; M. Berkooz and M. Rozali, "Near Hagedorn dynamics of NS fivebranes, or a new universality class of coiled strings," JHEP 05 (2000) 040, hep-th/0005047; D. Kutasov and D. A. Sahakyan, "Comments on the thermodynamics of little string theory," JHEP 02 (2001) 021, hep-th/0012258.

[14] S. S. Gubser and I. Mitra, "The evolution of unstable black holes in anti-de Sitter space," JHEP 08 (2001) 018, hep-th/0011127; H. S. Reall, "Classical and thermodynamic stability of black branes," Phys. Rev. D64 (2001) 044005, hep-th/0104071; S. S. Gubser and A. Ozakin, "Universality classes for horizon instabilities," hep-th/0301002. 